\title{
Study of heat pipe performance using an aqueous solution of n-Butanol
}

\author{
R. Senthilkumar ${ }^{1}$, S. Vaidyanathan ${ }^{2}$ and B. Sivaraman ${ }^{2}$ \\ ${ }^{1}$ Dept. of Mechanical Engg., Annamalai University, Annamalai Nagar - 608 002, Tamil Nadu, India. \\ rsklme@yahoo.com
}

\begin{abstract}
An experiment is conducted to enhance the heat-transport capability of capillary assisted heat pipes with an aqueous solution of $n$-Butanol, and its performance is compared with that of pure water. This study uses an aqueous solution of $\mathrm{n}$-Butanol having a positive surface tension gradient with temperature as a working medium with a view to accomplish an enhancement in the performance of capillary limit of heat pipe systems and operative stability. The test heat pipe is made of a copper tube with an external diameter of $22 \mathrm{~mm}$, and an inner diameter of $19.6 \mathrm{~mm}$. Stainless steel wick of wrapped screen structure has been used in the present study. The experiments were conducted with various heat inputs $(40 \mathrm{~W}, 60 \mathrm{~W} \& 80 \mathrm{~W})$ and the heat pipe was kept at different inclinations of $0^{\circ}, 45^{\circ}$ and $90^{\circ}$ with horizontal. The results show that the heat-transport capability of heat pipe with the aqueous solution of $n$-Butanol is higher than the water heat pipe.
\end{abstract}

Keywords: Heat pipe; aqueous solution of n-Butanol; surface tension; wrapped screen.

Introduction

The heat pipe is an effective device, which is capable of transferring large quantities of heat through relatively small cross-sectional areas and with very small temperature differences (Chi, 1976; Peterson, 1994; Faghri, 1995). Heat pipes consist of a sealed container with a small amount of a working fluid. The heat is transferred as latent heat energy by evaporating the working fluid in the evaporator zone and condensing the vapour in a cooling zone (condenser), the circulation is completed by return flow of the condensate to the evaporator zone through the capillary structure which lines the inner wall of the container. The heat pipes are more advantageous in heat recovery systems (Cannavilo et al., 1884; Littwin \& Willis, 1985), solar energy (Bloem, 1982), electronics cooling (Ivanova, 2006; Sonan, 2008), Audio amplification, liners for materials processing furnaces, Ocean thermal energy conversion, air craft cooling (Vochten \& Petre, 1973), geothermal conversion (Chandourene \& Gruss, 1987) and light water nuclear reactors (Kaminaga et al., 1997).

All of the heat pipes, including conventional heat pipes, flat heat pipes and micro heat pipes have common problem of heat transfer limitation. These limitations determine the maximum possible heat transfer rate for a particular heat pipe under normal working conditions. The various limitations of the heat pipe are continuum flow limit, frozen startup limit, viscous limit, sonic limit, entrainment limit, capillary limit, condenser limit and boiling limit (Chi, 1976; Faghri, 1995; Sonan, 2008). Among them the capillary limits and boiling limits are important for heat pipe design and operation. The surface tension is an important key factor for capillary limit along with boiling limit, sonic limit, entrainment limit, viscous limit and condenser limit. Surface tension of all pure liquids is normally decreasing with increase in temperature; indeed, the liquid is moved along the interface toward the cooler zone, which is the condenser zone. Since the working fluids that are currently available for heat pipes have negative surface-tension gradients with temperature, which are unfavourable for the spreading or re-wetting on a heated surface, and therefore, the operating temperature and the heat load of heat pipe systems are limited. The heat pipe systems also suffer from operational instability problems because of the characteristics of the negative surface tension gradient with temperature (Zhang, 2001). Obviously, the best way to overcome these problems is to explore a new working fluid, which has a positive surface-tension gradient with temperature in the operating-temperature region.

Generally, water is used as a working fluid for medium temperature applications due to its availability, cost, safety, easy to handle and high surface tension. But the surface tension of the water also decreases rapidly with increase in temperature. Vochten and Petre (1973) experimentally revealed that the surface tension of aqueous solutions of alcohols, with chain lengths longer than four carbon atoms, have a positive gradient with temperature when the temperature exceeds a certain value. The stable working fluid circulation in a heat pipe is achieved through the capillary pressure head developed by the wick structure. The maximum capillary head developed in the heat pipe must be more than the various pressure losses along the vapour-liquid path. i.e. liquid pressure drop, Pressure drop in wick, vapour pressure drop and pressure drop in the liquid due to the effect of gravitational force.

In the proposed method, heat pipe of copper container with stainless steel wick material and aqueous solutions as working fluid have been analyzed. $1 \times 10^{-3} \mathrm{~mol} / \mathrm{l}$ of $\mathrm{n}$ Butanol is added to the pure water and this solution is used as working fluid for the heat pipe. Wrapped screen wick structure with two layers of 40 mesh size was used
Research article

CIndian Society for Education and Environment (iSee)
"Heat transport"

http://www.indjst.org
Senthilkumar et al. Indian J.Sci.Technol. 
Fig. 1. Schematic diagram of experimental set up

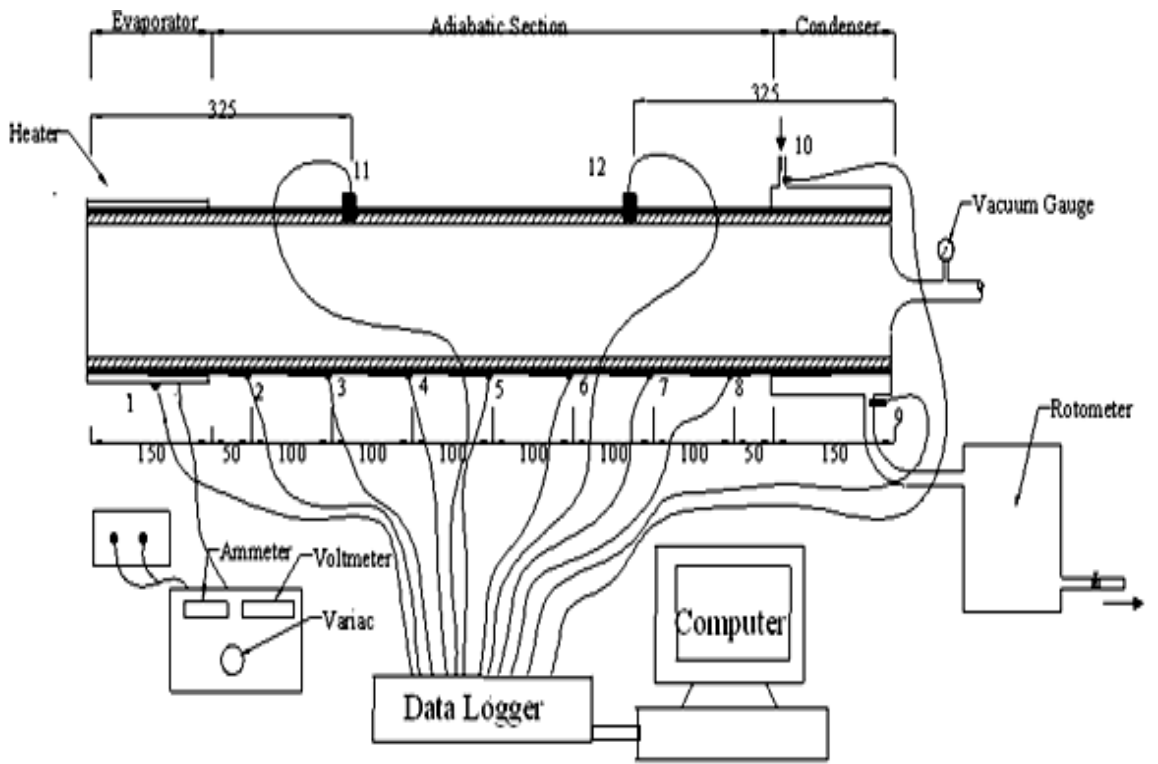

in the heat pipe. The experiments were conducted for various flow rates in condenser section $(0.06,0.08$, and 0.1 $\mathrm{kg} / \mathrm{min}$ ) and for various inclinations of heat pipe to the horizontal $\left(0^{\circ}, 45^{\circ} \&\right.$ $\left.90^{\circ}\right)$ with different heat inputs $(40,60$ \& $80 \mathrm{~W})$. A thermal resistance $(R)$ is also calculated for both the heat pipes using the relation

$$
R=\frac{Q}{\Delta T}
$$

Where $Q$ is the heat supply, $\Delta T$ is the temperature difference between the evaporator and condenser. The results obtained with the aqueous solution of n-Butanol were compared with water heat pipe.

\section{Experimental setup}

Table 1. Specifications of heat pipe

\begin{tabular}{|l|l|}
\hline \multicolumn{1}{|c|}{ Specifications } & Dimensions \\
\hline Outside diameter, $\mathrm{m}$ & 0.022 \\
\hline Inside diameter, $\mathrm{m}$ & 0.0196 \\
\hline Evaporator length, $\mathrm{m}$ & 0.15 \\
\hline Condenser length, $\mathrm{m}$ & 0.15 \\
\hline Adiabatic length, $\mathrm{m}$ & 0.70 \\
\hline Total length, $\mathrm{m}$ & 1.00 \\
\hline Wick mesh size, $\mathrm{m}$ & 1600 \\
\hline Wick porosity & 0.769 \\
\hline No. of layers of wick & 2 \\
\hline Wick permeability, $\mathrm{m}^{2}$ & $2.213 \times 10^{-9}$ \\
\hline Capillary Limit, $\mathrm{W}$ & 108.8 \\
\hline Sonic Limit, $\mathrm{W}$ & 83852.5 \\
\hline Entrainment Limit, $\mathrm{W}$ & 5553.2 \\
\hline Boiling Limit, $\mathrm{W}$ & 711.6 \\
\hline
\end{tabular}

Result and discussions

Efficiency comparison of the aqueous solution of $n$-Butanol with water

Fig. 2-4 show the bar charts for 40 $\mathrm{W}, 60 \mathrm{~W} \& 80 \mathrm{~W}$ heat input vs. flow rate for $0^{\circ}, 45^{\circ} \& 90^{\circ}$ inclinations in the heat pipe with the aqueous solution of n-Butanol and water. The efficiency of heat pipe with the aqueous solution of n-Butanol is higher than the heat pipe with water for all heat inputs, flow rate and inclinations, except for the case of $90^{\circ}$ inclinations for $60 \mathrm{~W}$ and $90 \mathrm{~W}$ heat input, where the efficiency of the heat pipe with the aqueous solution of $\mathrm{n}$-Butanol is less than the water. It is due to the fact that, at higher temperature the surface tension of the

The schematic diagram of the experimental setup is shown in Fig.1. The specifications of heat pipe are tabulated in Table 1. Heat input was given at the evaporator section using an electric strip attached to it with proper electrical insulation and heater was energized with an AC supply through a variac. The desired heat input was supplied to the evaporator end of the heat pipe by adjusting the variac. Water jacket at the condenser end was used to remove the heat from the pipe. The heat pipe has the ability to transfer the heat through the internal structure. As a result, sudden rise in wall temperature occurs which could damage the heat pipe if the heat was not released at the condenser properly. Therefore, before heat was supplied to the evaporator, the cooling water was first circulated through the condenser jacket. The power input was gradually raised aqueous solution of $\mathrm{n}$-Butanol also decreases. Moreover, the gravitational force that assist the flow of working fluid back to the evaporator may accelerate the process which may hinder the heat transfer process at the condenser end and the fluid might have returned to the evaporator section with higher temperature end. This may be the reason why the performance of heat pipe deteriorates when the inclination was increased. This problem may not arise if the condenser section is at the base and evaporator at the top and only the capillary forces the liquid to move upward towards the evaporator.

\section{Heat transfer coefficient in Condenser side for the aqueous solution of $n$-Butanol} transfer coefficient for $0^{\circ}, 45^{\circ} \& 90^{\circ}$ inclinations and $40 \mathrm{~W}$, $60 \mathrm{~W} \& 80 \mathrm{~W}$ of heat input in the heat pipe. From the
Fig. 5-7 show the bar chart for heat input vs. heat
Research article

CIndian Society for Education and Environment (iSee)
"Heat transport" http://www.indjst.org
Senthilkumar et al. Indian J.Sci.Technol. 
Fig. 2. Efficiency comparison of heat pipes for $0^{\circ}$ inclination

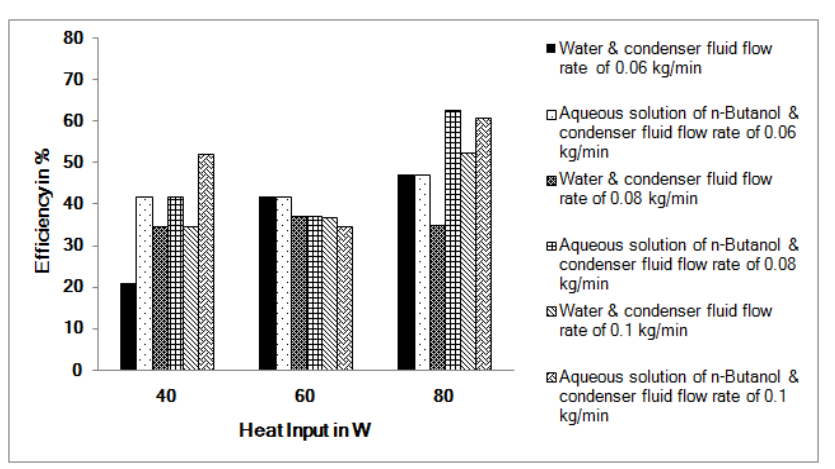

Fig. 4. Efficiency comparison of heat pipes for $90^{\circ}$ inclination

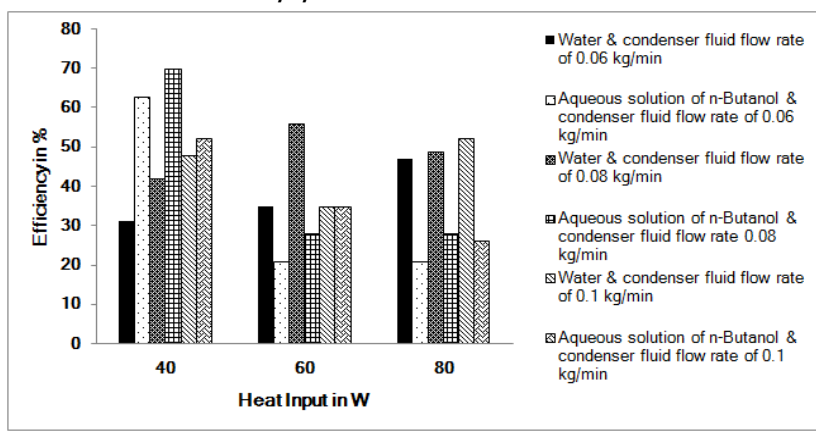

Fig. 6. Variation of heat transfer coefficient in the condenser side for $45^{\circ}$ inclination

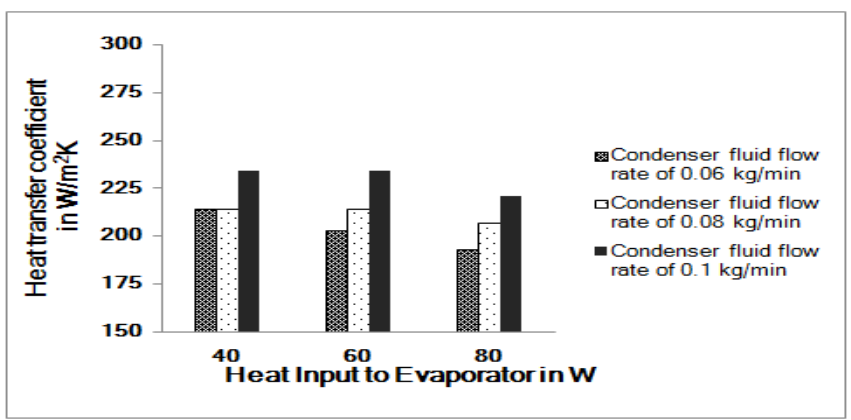

Fig. 8. Overall heat transfer coefficient comparison for $0^{\circ}$ inclination

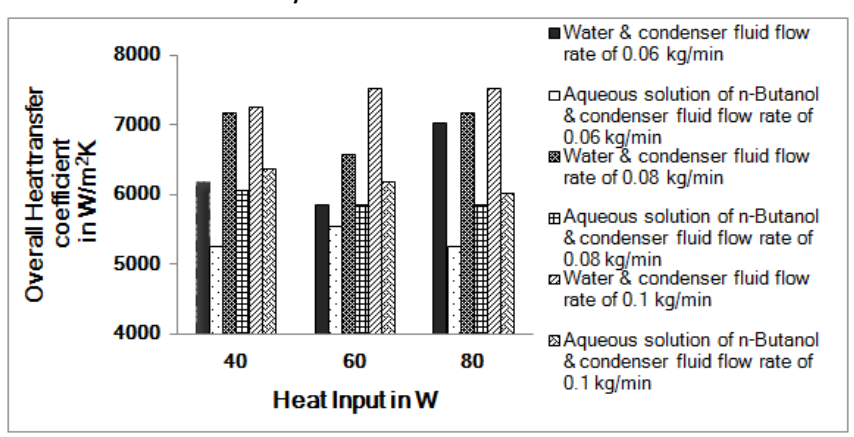

Vol. 3 No. 6 (June 2010)

ISSN: 0974- 6846

Fig. 3. Efficiency comparison of heat pipes for $45^{\circ}$ inclination

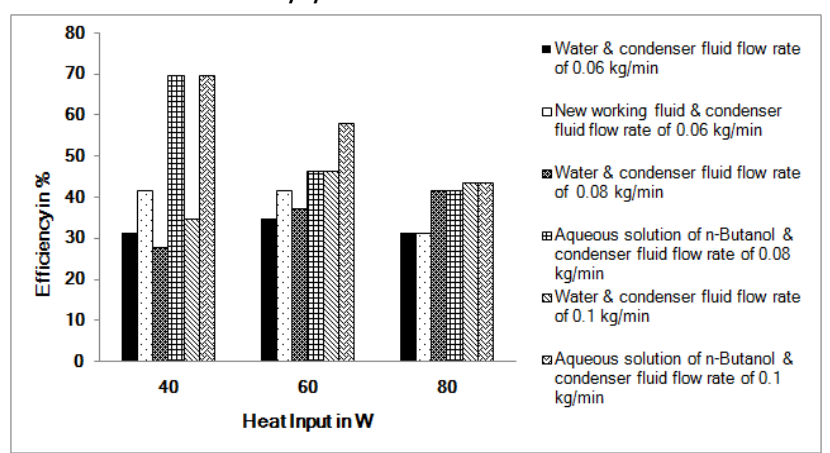

Fig. 5. Variation of heat transfer coefficient in the condenser side for $0^{\circ}$ inclination

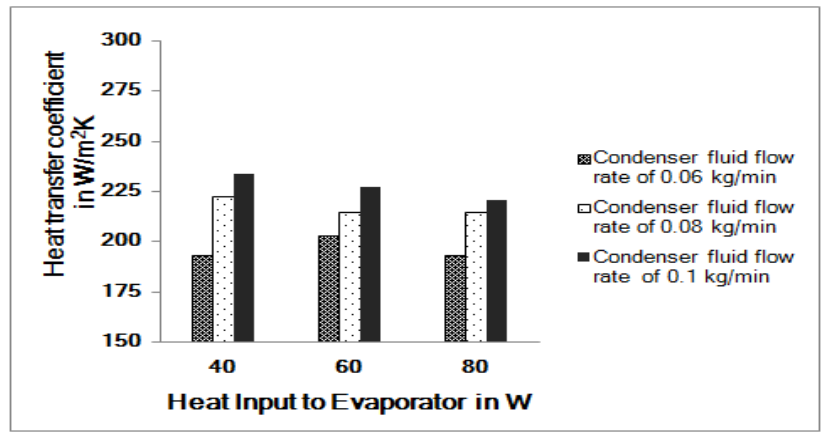

Fig. 7. Variation of heat transfer coefficient in the condenser side for $90^{\circ}$ inclination

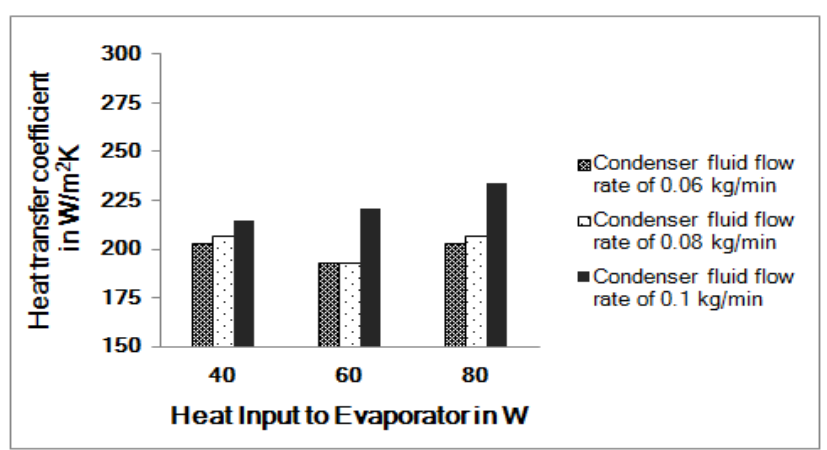

Fig. 9. Overall heat transfer coefficient comparison for $45^{\circ}$ inclination

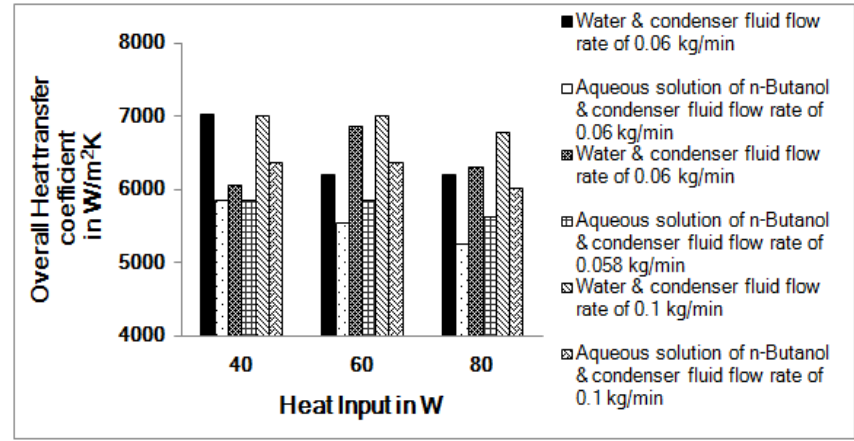

Research article

CIndian Society for Education and Environment (iSee)
"Heat transport" http://www.indjst.org
Senthilkumar et al. Indian J.Sci.Technol. 
Fig. 10. Overall heat transfer coefficient comparison for $90^{\circ}$ inclination

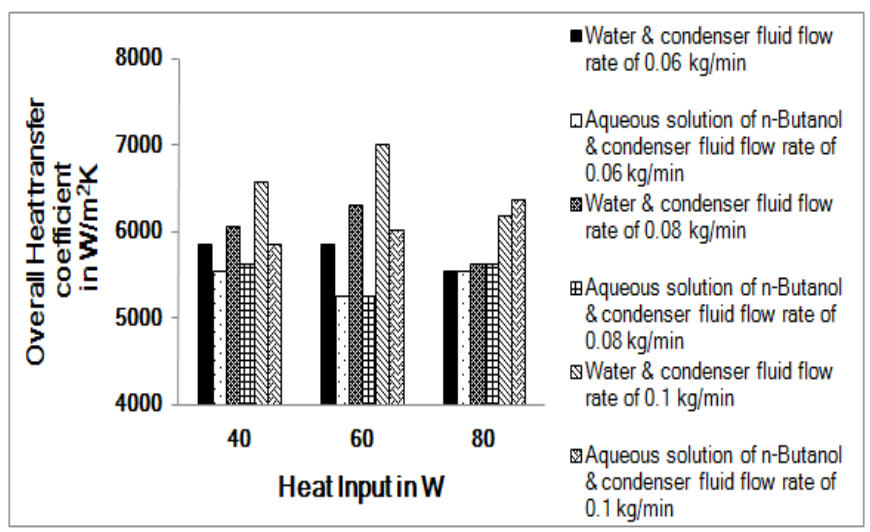

Fig. 12. Thermal resistance comparison for $45^{\circ}$ inclination

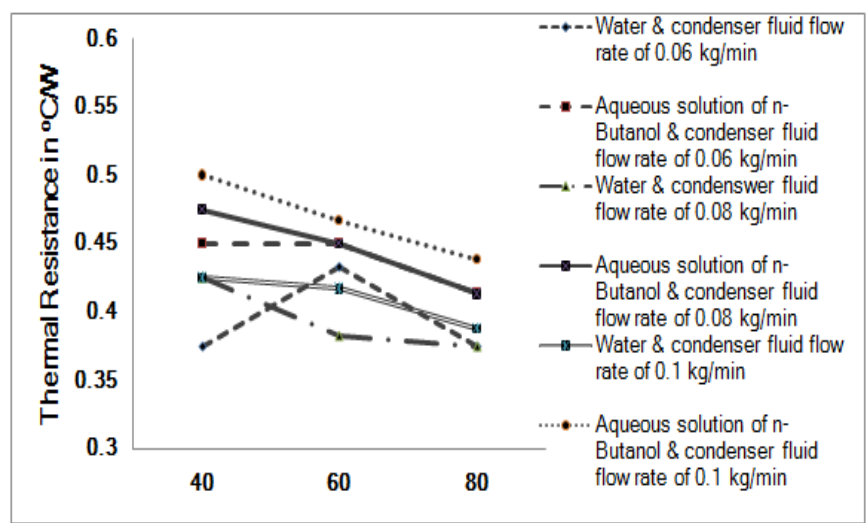

above figures, it is shown that the heat transfer coefficient in condenser section increases with the increase in both the flow rate and heat pipe inclination. Also it is seen that the heat transfer coefficient is high when the heat pipe is kept at vertical position. The average value of heat transfer coefficient in the condenser side is around 220 $\mathrm{W} / \mathrm{m}^{2}{ }^{\circ} \mathrm{C}$.

\section{Overall heat transfer coefficient}

The overall heat transfer coefficients for the various conditions are studied and evaluated for all the heat inputs, inclinations and flow rates. Fig. 8-10 show the bar chart for heat input vs. overall heat transfer coefficient for $0^{\circ}, 45^{\circ} \& 90^{\circ}$ inclinations and $40 \mathrm{~W}, 60 \mathrm{~W} \& 80 \mathrm{~W}$ of heat input in the heat pipe for water and the aqueous solution of $n$-Butanol. When water is used as the working fluid, the overall heat transfer coefficient has a value ranging from 5500 to $7200 \mathrm{~W} / \mathrm{m}^{2 \circ} \mathrm{C}$. But, when the aqueous solution of n-Butanol is used under similar conditions, the overall heat transfer coefficient reaches the values ranging from 5200 to $6300 \mathrm{~W} / \mathrm{m}^{2 \circ} \mathrm{C}$. The reason for this reduction is due to the increase in surface temperature of the aqueous solution of $n$-Butanol heat pipe.
Fig. 11. Thermal resistance comparison for $\sigma^{\circ}$ inclination

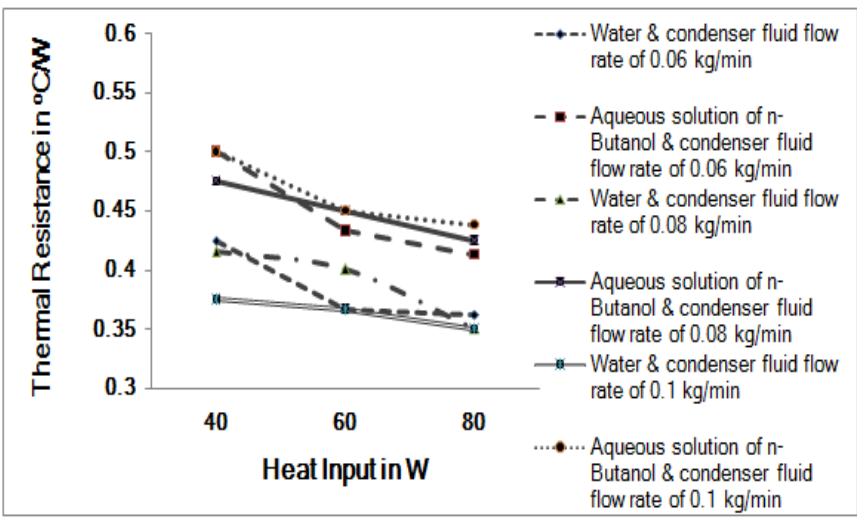

Fig. 13. Thermal resistance comparison for $90^{\circ}$ inclination

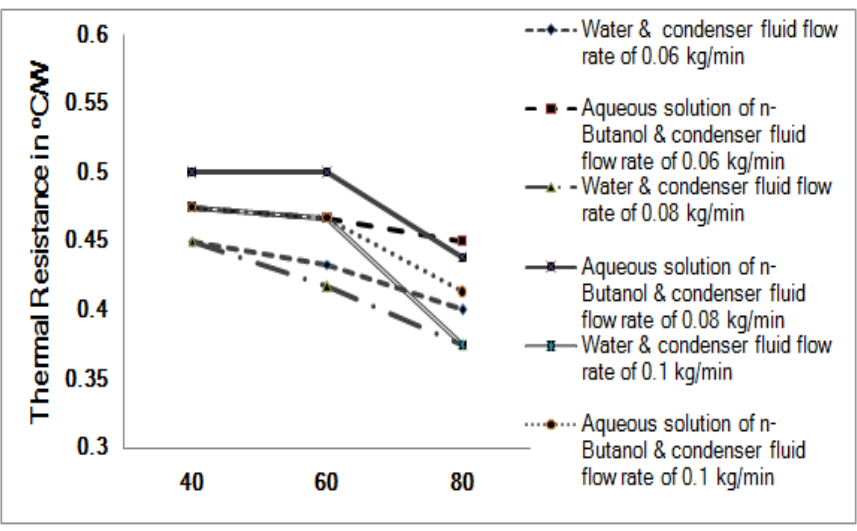

\section{Effect of thermal resistance}

The thermal resistance of the heat pipe is studied for various conditions and evaluated for all heat inputs, inclinations and flow rates. Fig.11-13 show the variation of thermal resistance with respect to heat input for $0^{\circ}, 45^{\circ}$ $\& 90^{\circ}$ inclinations and $40 \mathrm{~W}, 60 \mathrm{~W} \& 80 \mathrm{~W}$ of heat input in the heat pipe for water and the aqueous solution of $n$ Butanol. The thermal resistance of the aqueous solution of $n$-Butanol heat pipe is higher than the water heat pipe for all inclinations and flow rates. At $40 \mathrm{~W}$ heat input, the thermal resistance is almost same which is in the order of $0.5^{\circ} \mathrm{C} / \mathrm{W}$, irrespective of flow rate and the angle of inclination.

\section{Conclusion}

- The experiments show that the efficiency of the heat pipe is a function of flow rate, inclination angle and heat input.

- The efficiency and heat transfer coefficient are considerably increased for aqueous solution of n-Butanol.
Research article

CIndian Society for Education and Environment (iSee)
Senthilkumar et al. Indian J.Sci.Technol. 
- The surface temperature distribution along the adiabatic section of the heat pipe is within the permitted limit.

- The heat pipe with the aqueous solution of n-Butanol gives the better performance at $45^{\circ}$ Inclination. The efficiency of the aqueous solution of n-Butanol heat pipe is more for $45^{\circ}$ inclinations and at a flow rate of 0.1 $\mathrm{kg} / \mathrm{min}$.

- Heat transfer coefficient in condenser section is high when the heat pipe is kept at vertical position. The average value of heat transfer coefficient in condenser side is around $220 \mathrm{~W} / \mathrm{m}^{2}{ }^{\circ} \mathrm{C}$.

\section{References}

1. Bloem H, De-Grijis JC and Devaan RLC (1982) An evacuated tubular solar collector incorporating a heat pipe. Philips Tech. Rev. 40, 181-191.

2. Cannavilo $M$, Caaarosa $C$, Latrofa $E$, Martorano $L$ and Reale F (1981) Gravity heat pipes as geothermal convectors. Proc. $4^{\text {th }}$ Int. Heat Pipe Conf. p: 759.

3. Chandourene S and Gruss A (1987) Theoretical and experimental study of high temperature heat pipe heat exchanger application to $1300 \mathrm{KW}$ respirator. $6^{\text {th }}$ Int. heat pipe Conf. Grenoffle, France.

4. Chi SW (1976) Heat pipe theory and practice. McGraw-Hill, Washington.

5. Faghri A (1995) Heat pipe science and technology. Taylor \& Francis, Washington.

6. Ivanova M, Avenas $Y$, Schaeffer $C$, Dezord JB and Schulz-Harder J (2006) Heat pipe integrated in direct bonded copper (DBC) technology for cooling of power electronics packaging. IEEE Trans. Power Electronics. 21 (6), 1541.

7. Kaminaga $F$, Hashimoto $H$, Feroz $C$, Goto $K$ and Masumura K (1997) Heat transfer characteristics of evaporation and condensation in a two-phase closed thermosyphon, Proc. $10^{\text {th }}$ Int. Heat Pipe Conf., Germany.

8. Littwin DA and Willis DB (1985) The use of heat pipes to conserve energy in petroleum refineries. Energy Process, 5, 198-202.

9. Peterson (1994) An introduction to heat pipes, Wiley \& Sons.

10.Sonan R, Harmand S, Pellé J, Leger D and Fakès $M$ (2008) Transient thermal and hydrodynamic model of flat heat pipe for the cooling of electronics components. Int. J. Heat Mass Transfer. 51, 60066017.

11.Vochten R and Petre G (1973) Study of the heat of reversible adsorption at the air-solution interface, II. Experimental determination of the heat of reversible adsorption of some alcohols. J. Cillid Interface. Sci. $42,320$.

12.Zhang N (2001) Innovative heat pipe systems using a new working fluid, Inl. Comm. Heat Mass Transfer. 28(8), 1025-1033. 